\title{
Efficacy of Galantamine on Cognition in Mild-to-Moderate Alzheimer's Dementia after Failure to Respond to Donepezil
}

\author{
Tae-Young Hwang ${ }^{1,2}$, Inn-Sook Ahn³, Seonwoo Kim4, and Doh Kwan Kim ${ }^{1,3 凶}$ \\ ${ }^{1}$ Department of Psychiatry, Samsung Medical Center, Sungkyunkwan University School of Medicine, Seoul, Republic of Korea \\ ${ }^{2}$ Department of Psychiatry, Jeonbuk Provincial Maeumsarang Hospital, Wanju, Republic of Korea \\ ${ }^{3}$ Center for Clinical Research, Samsung Biomedical Research Institute, Seoul, Republic of Korea \\ ${ }^{4}$ Biostatistics Unit, Samsung Biomedical Research Institute, Seoul, Republic of Korea
}

Objective This study compares the efficacy of the cholinesterase inhibitor (ChEI) galantamine on cognition in patients with mild-to-moderate Alzheimer's dementia (AD) who were either naïve to ChEI drugs or who had failed a trial of the ChEI donepezil.

Methods Outpatients with AD were sequentially referred for screening and enrollment. Current outpatients who had taken donepezil for at least 6 months without demonstrated efficacy on cognition were switched to galantamine (switched group). New outpatients with no ChEI prescription history were classified as the naïve group and were given galantamine. The primary outcome measures for the betweengroup comparison were response rate on cognition at 26 and 52 weeks (categorical) and change on the Korean version of the Alzheimer's Disease Assessment Scale-cognitive subscale (dimensional). Secondary cognitive outcomes were measured using the subset of frontal executive function and the Korean Mini-Mental State Examination.

Results Seventy outpatients were enrolled and 66 were analyzed by Intent-to-treat (ITT). There were 42 cases in the naïve group and 24 in the switched group. Response rates did not differ at 26 weeks ( $71.4 \%$ naïve vs. $58.3 \%$ switched; $p=0.277$ ) or at 52 weeks ( $59.5 \%$ naïve vs. $41.6 \%$ switched; $\mathrm{p}=0.162$ ). No significant differences were observed in the pattern of change over the 52 weeks on the primary and secondary cognitive scales.

Conclusion As the efficacy of galantamine on cognition was not inferior in the switched group compared to that in the naïve group, switching ChEI drugs is clinically feasible for non-responding patients with mild-to-moderate AD.

Psychiatry Investig 2016;13(3):341-348

Key Words Alzheimer's dementia, Donepezil, Galantamine, Switching, Cognition, Efficacy.

\section{INTRODUCTION}

Cholinesterase inhibitors (ChEIs) are currently recommended as the first-line treatment for patients with mild-to-moderate Alzheimer's dementia (AD). ${ }^{1,2}$ Most clinical trials suggest that there is little difference in efficacy among available ChEI drugs. ${ }^{3,4}$ In practice, clinicians discontinue a ChEI when they see no evidence of therapeutic efficacy, and few studies have tested whether switching nonresponsive patients to another ChEI is beneficial. In contrast, such guidance is available for

Received: March 5, 2015 Revised: July 31, 2015

Accepted: September 28, 2015 Available online: February 17, 2016

$\triangle$ Correspondence: Doh Kwan Kim, MD, PhD

Department of Psychiatry, Samsung Medical Center, Sungkyunkwan University School of Medicine, 81 Irwon-ro, Gangnam-gu, Seoul 06351, Republic of Korea Tel: +82-2-3410-3582, Fax: +82-2-3410-0941, E-mail: paulkim@skku.edu

(a) This is an Open Access article distributed under the terms of the Creative Commons Attribution Non-Commercial License (http://creativecommons.org/licenses/bync/3.0) which permits unrestricted non-commercial use, distribution, and reproduction in any medium, provided the original work is properly cited. patients with depression or migraine where multiple therapeutic options exist. ${ }^{5-8}$ Differences in the pharmacokinetic and pharmacodynamic profiles among ChEIs may provide a clinical benefit after switching to another $\mathrm{ChEI},{ }^{9-11}$ whereas previous failure to respond to one ChEI may predict failure to respond to a second ChEI based on pharmacodynamics. Our study is aimed at clarifying this issue. We compared the efficacy of galantamine on cognition in a group that was naive to all ChEI drugs (naïve group) and in a second group who failed to respond to donepezil and was switched to galantamine (switched group).

\section{METHODS}

\section{Study design}

This 52-week, open-label, prospective, single-blinded, naturalistic observational study was conducted on outpatients visiting the Clinical Trials Program of the Samsung Medical 
Center Geropsychiatry Clinics (Seoul, Korea). Both old and new outpatients with $\mathrm{AD}$ were sequentially interviewed and referred for screening and enrollment. Current outpatients who had been taking donepezil and shown lack or loss of efficacy on cognition on subsequent regular evaluations were identified as candidates for the switched group. Lack or loss of efficacy on cognition for these cases was based on the same definition of response as in this study, i.e., improvement or no deterioration on the total score of the primary cognitive outcome measure at the time of enrollment. New outpatients were identified as candidates for the naïve group. All outpatients received a diagnosis of mild-to-moderately severe $\mathrm{AD}$, and all were treated with galantamine. The trial duration was 52 weeks, consistent with the goal of assessing the long-term benefit of galantamine. ${ }^{12,13}$ An open label design without a placebo control group was used for ethical reasons in view of trial length. ${ }^{12,14}$ As an alternative to placebo control groups, we employed the Stern equation to impute the predicted natural decline in scores. ${ }^{15}$ Thus, we could verify that galantamine was efficacious before proceeding to the group comparison (naïve vs. switched).

\section{Subjects}

We identified 93 consecutive outpatients with $\mathrm{AD}$ as candidates for screening and enrollment. Twenty-three were excluded due to withdrawal of consent $(n=16)$ and failure to meet the severity criteria $(n=7)$. Accordingly, 70 outpatients with $\mathrm{AD}$ were enrolled. After enrollment, four outpatients did not satisfy the intent-to-treat (ITT) criteria as detailed in the Data Analysis section. Thus, 66 patients were analyzed in the ITT population ( $n=42$ in the naïve group and $n=24$ in the switched group). Fifty-one of the 66 ITT patients (77\%) completed the 52 week study duration, and no significant betweengroup difference was detected in the drop-out rate at 52 weeks ( $n=11$ in naive group and $n=4$ in switched group; Fisher exact test, $\mathrm{p}=0.543$ ). Figure 1 shows the flow chart of patients through the study and the reasons for non-completion.

All patients met the diagnostic criteria for probable $\mathrm{AD}$ according to the National Institute of Neurological and Communicative Disorders and Stroke-Alzheimer's Disease and Related Disorders Association (NINCDS-ADRDA), ${ }^{16}$ with a history of gradual onset and progressive cognitive decline over at least 6 months. The severity criterion was a Korean

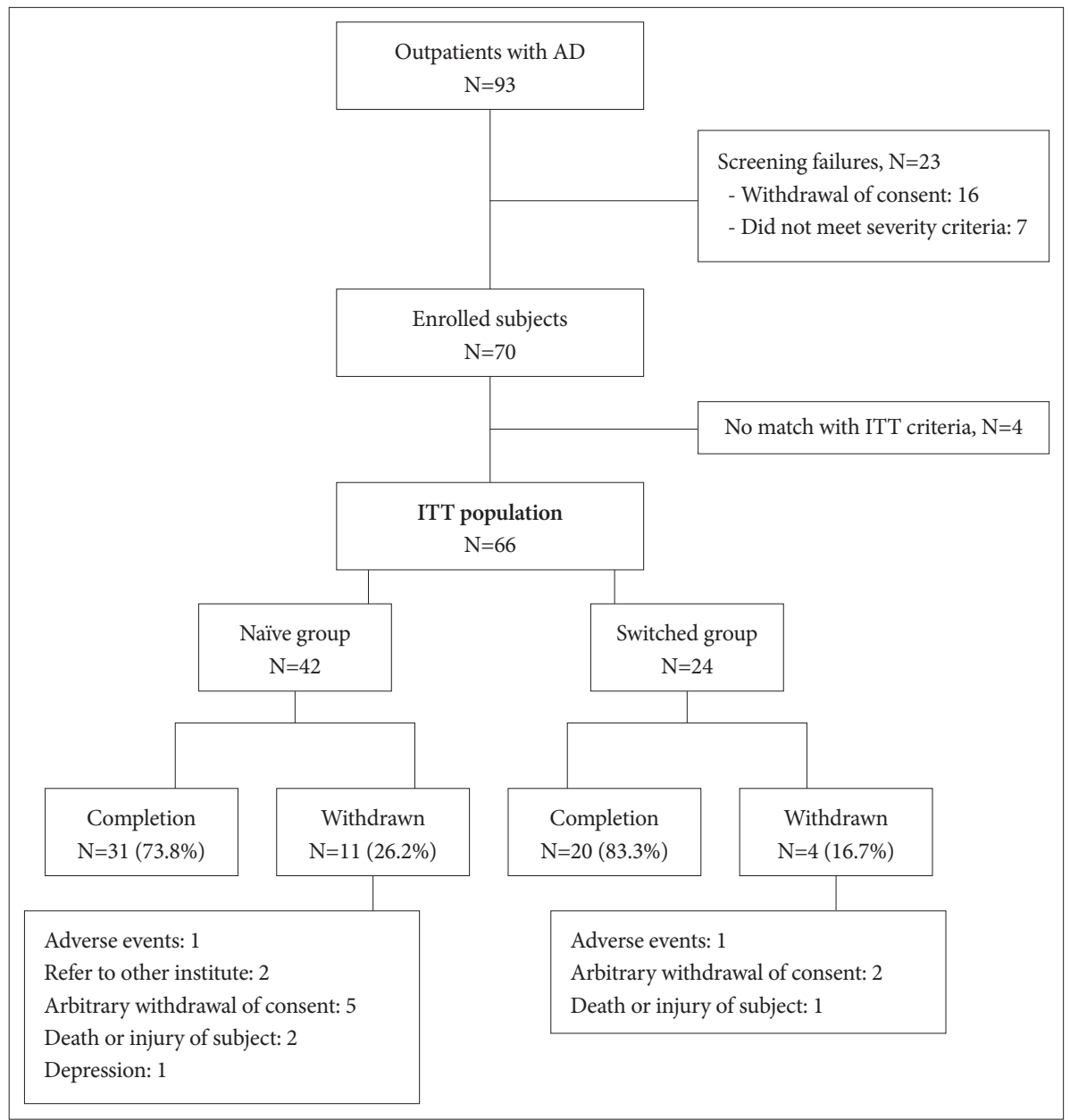

Figure 1. Flow chart of the study. Study completion refers to the endpoint of the study at 52 weeks. AD: Alzheimer's dementia, ITT: intent-to-treat. 
Mini-Mental State Examination (K-MMSE) score of 10-26 points. ${ }^{17}$ Additionally, the Clinical Dementia Rating Sum of Boxes scale (CDR-SB) was used for clinical staging of dementia. ${ }^{18}$ Patients were excluded if they had evidence of other neurodegenerative diseases (Parkinson's disease, Huntington's disease, Down's syndrome, or Creutzfelt-Jacob's disease), a psychiatric disorder, severe behavioral disturbances that required intensive care and psychotropic medications, and cerebral injuries induced by trauma, hypoxia, and/or ischemia. Additional exclusion criteria included a history of a seizure disorder and concomitant medical diseases that needed to be treated promptly. Each patient had to have a caregiver who could administer the patient's medication and report on behavioral and functional items. This study was approved by the local Institutional Review Board (IRB) at Samsung Medical Center, and written informed consent was obtained from both the caregivers and the patients. The study was registered (NCT01029132) at ClinicalTrials.gov.

The initial evaluation included a thorough history-taking, evaluation of vital signs, physical and neurological examinations, laboratory tests, electrocardiogram (ECG), and brain magnetic resonance imaging. Patient interviews were conducted at 1 and 4 weeks after commencing galantamine to adjust the dosage and evaluate adverse events. If there were no significant changes or side effects, the next evaluation was scheduled to take place 4 weeks later. The physical, neurological, and side-effects evaluations were performed by experienced geropsychiatrists. Blinded neuropsychologists carried out the independent neuropsychological evaluations at baseline, 4, 13, 26, 39, and 52 weeks.

Each participant received galantamine at a dose of $8 \mathrm{mg} /$ day ( $4 \mathrm{mg}$ bid) over the first 4 weeks. The dose was increased by $8 \mathrm{mg} /$ day at 4 -week intervals, up to a maximum daily dose of $24 \mathrm{mg}$ /day (12 mg bid). The dosage was flexibly maintained at $16-24 \mathrm{mg} /$ day, according to the patient's tolerance. As the switched group comprised outpatients who were already tolerating a ChEI (donepezil) with no significant problems, the switch to galantamine was made without a wash-out period. These switching patients stopped donepezil and started galantamine the next day. ${ }^{19,20}$ Psychotropic medications other than galantamine were not allowed, except benzodiazepines only as a short-term adjunctive for insomnia.

The tolerability and safety of galantamine were regularly assessed throughout the study, and vital signs, physical examination, laboratory tests, and ECG were included. The details of the adverse events experienced by the patients, such as specific symptoms and the causal relationship of each adverse event were evaluated at each visit through clinician inquiry and observation, along with adverse event reports provided by the patients and their caregivers. Adverse events were re- corded using the UKU side effect rating scale. ${ }^{21}$

\section{Cognitive outcome measures}

The categorical primary outcome measure was response rate on cognition at 26 and 52 weeks. We defined response as improvement or no deterioration on the total Korean version of the Alzheimer's Disease Assessment Scale-cognitive subscale (ADAS-cog-K) score compared with baseline. ${ }^{22,23}$ Nonresponse was defined as a worsening of the ADAS-cog-K score. ADAS-cog-K is a Korean translated version of the original ADAS-cog, in which frontal executive function, one of the cognitive subdomains, is not satisfactorily covered. ${ }^{24,25}$ ADAS-cog$\mathrm{K}$ is a reliable and valid instrument for diagnosing $\mathrm{AD}$ and evaluating severity and is composed of three subdomain subscales, i.e., memory (score 0-35), language (score 0-25), and praxis (score $0-10$ ). Higher scores on the ADAS-cog-K (total score range, 0-70) indicate greater cognitive impairment.

The dimensional primary outcome measure was magnitude of change on the ADAS-cog-K, in which change was the observed score minus the baseline score at each evaluation point (i.e., 4, 13, 26, 39, and 52 weeks).

In addition to the ADAS-cog-K, a secondary measure of cognitive outcome was obtained using a subset of tests for evaluating frontal executive function developed by the Alzheimer's Disease Cooperative Study. ${ }^{26}$ This subset comprised the trail making test-parts $\mathrm{A}$ and $\mathrm{B},{ }^{27}$ digit span, ${ }^{28}$ and category fluency (animal and supermarket items), ${ }^{29}$ with a summed scoring range of $0-25$.

An additional secondary cognitive outcome was change on the K-MMSE, ${ }^{30}$ which is the Korean version of the original MMSE, ${ }^{31}$ which has been validated for screening dementia and has a score range of $0-30$.

\section{Statistical analysis}

\section{Sample size and power analysis}

The results of a previous clinical trial with galantamine were considered to determine the minimum number of participants for this study, in which the galantamine group taking $16 \mathrm{mg} /$ $\mathrm{d}$ showed a decrease of $3.3 \pm 4.2$ [mean \pm standard deviation (SD)] on the original ADAS-cog score, compared to the placebo group after 21 weeks. ${ }^{32}$

If previous failure to respond to donepezil generalizes to other ChEIs, then a switch to galantamine will confer no benefit. If the previous failure does not generalize, then the expected change in ADAS-cog score would be 3.3 44.2 (mean \pm SD). Accordingly, sample sizes of 42 in the naïve group and 24 in the switched group would achieve $86.66 \%$ power, indicating sensitivity $>85 \%$ that the differences on the ADAScog-K between the two groups would be detected. 


\section{Data analysis}

The comparative analyses were based on the ITT population, which included all subjects who received at least one dose of study medication, provided baseline data, and had at least one post-baseline efficacy assessment. The last observation carried forward (LOCF) method was used to analyze patients who did not complete the study. Student's t-test or the MannWhitney test was used for continuous variables, and the chisquare test was used for categorical variables to investigate the between-group differences in baseline characteristics (Table 1).

Before comparing the naïve and switched groups, we assessed the efficacy of galantamine on cognition in the total galantamine group ( $n=66$ ), using the Stern equation to impute a placebo group. Paired t-tests and Wilcoxon's signed-rank tests with a Bonferroni's correction were used for this comparison.

The Stern equation is as follows: predicted ADAS-cog-K score at time $\mathrm{T}=-6.039689+1.329485 \mathrm{xi}-0.005392 \times 2 \mathrm{i}+$ $(0.031974+0.036652 \times \mathrm{xi}-0.000473634 \mathrm{x} 2 \mathrm{i}) \mathrm{T}$, where $\mathrm{T}$ is months from baseline, and xi is baseline ADAS-cog-K score for each participant. $^{15}$
Student's t-test and the Mann-Whitney test with Bonferroni's correction were used to compare changes on each scale between the naïve and switched groups at each evaluation point $(4,13,26,39$, and 52 weeks). The Bonferroni correction was used to control the increase in type I error due to multiple comparisons between the naïve and switched groups on changes in each scale over the 52 weeks. Furthermore, the difference of pattern of change over 52 weeks on each scale between the two groups was compared using repeated-measures analysis of variance (ANOVA). Spearman's partial correlation analysis was applied to adjust the between-group difference in the duration of illness.

SPSS ver. 19 for Windows software (SPSS Inc., Chicago, IL, USA) was used for the analysis. A two-sided $\mathrm{p}<0.05$ was considered significant.

\section{RESULTS}

\section{Baseline demographic and clinical characteristics}

There were 1.5 times more female subjects $(n=40,60.6 \%)$

Table 1. Demographic and clinical characteristics of the ITT population

\begin{tabular}{|c|c|c|c|c|}
\hline Characteristics & Naïve group $(\mathrm{N}=42)$ & Switched group $(\mathrm{N}=24)$ & Statistics & $\mathrm{p}$ \\
\hline \multicolumn{5}{|l|}{ Sex, N (\%)* } \\
\hline Female & $27(64.2)$ & $13(54.1)$ & $\chi^{2}=0.655$ & 0.418 \\
\hline Male & $15(35.8)$ & $11(43.9)$ & & \\
\hline Age $(\text { year })^{\dagger}$ & $73.59(9.43)$ & $72.20(8.50)$ & $Z=-0.801$ & 0.422 \\
\hline Education(year) ${ }^{\dagger}$ & $9.26(5.59)$ & $9.00(5.65)$ & $Z=-0.081$ & 0.935 \\
\hline Duration of illness (month) ${ }^{\dagger}$ & $17.04(12.53)$ & $37.62(21.01)$ & $Z=3.931$ & $<0.001$ \\
\hline CDR-SB score ${ }^{\ddagger}$ & $6.28(3.01)$ & $7.00(3.41)$ & $\mathrm{t}=-0.883$ & 0.381 \\
\hline ADAS-cog-K score ${ }^{\dagger}$ & $27.52(9.57)$ & $28.29(8.17)$ & $\mathrm{Z}=0.634$ & 0.526 \\
\hline Subset of memory ${ }^{\ddagger}$ & $19.26(5.95)$ & $19.70(5.04)$ & $\mathrm{t}=-0.310$ & 0.758 \\
\hline Subset of language ${ }^{\dagger}$ & $5.83(3.18)$ & $6.00(3.38)$ & $\mathrm{Z}=0.046$ & 0.962 \\
\hline Subset of praxis ${ }^{\dagger}$ & $2.42(1.92)$ & $2.58(1.74)$ & $Z=0.591$ & 0.554 \\
\hline Subset of frontal executive function ${ }^{\dagger}$ & $14.30(3.31)$ & $14.12(3.43)$ & $\mathrm{Z}=0.006$ & 0.994 \\
\hline K-MMSE score ${ }^{\dagger}$ & $19.00(4.24)$ & $18.25(4.36)$ & $Z=-0.803$ & 0.422 \\
\hline
\end{tabular}

Continuous variables are shown as mean (SD). ${ }^{*}$ chi-square test, ${ }^{\dagger}$ Mann-Whitney test, ${ }^{\ddagger}$ Student’s t-test. ITT: intent-to-treat, SD: standard deviation, CDR-SB: Clinical Dementia Rating Sum of Box score, K-MMSE: Korean version of Mini-Mental State Examination, ADAS-cog-K: Alzheimer's Disease Assessment Scale-cognitive subscale, Korean version

Table 2. Comparison of changes on the ADAS-cog-K over 52 weeks between the imputed placebo and total galantamine groups

\begin{tabular}{lcccc}
\hline \multicolumn{1}{c}{ Time of evaluation } & Imputed placebo group $(\mathrm{N}=66)$ & Total galantamine group $(\mathrm{N}=66)$ & Statistics & $\mathrm{p}$ \\
\hline 4 weeks* & $-0.646(0.083)$ & $1.136(4.797)$ & $\mathrm{t}=3.015$ & 0.037 \\
13 weeks & & $-1.940(0.250)$ & $0.833(5.211)$ & $\mathrm{S}=645.5$ \\
26 weeks* & $-3.880(0.501)$ & $0.863(5.798)$ & $\mathrm{t}=6.558$ & 0.001 \\
39 weeks $^{\dagger}$ & $-5.821(0.752)$ & $-0.106(5.928)$ & $\mathrm{S}=886.5$ & 0.001 \\
52 weeks* & $-7.761(1.002)$ & $-0.787(5.856)$ & $\mathrm{t}=9.421$ & 0.001 \\
\hline
\end{tabular}

Data shown at each evaluation time are mean (SD) differences between baseline and current score on the ADAS-cog-K. Scores for the imputed placebo group were estimated using the Stern equation applied to baseline scores on the ADAS-cog-K. *paired t-test with Bonferroni's correction, ${ }^{\dagger}$ Wilcoxon's signed-rank test with Bonferroni's correction. SD: standard deviation, ADAS-cog-K: Alzheimer's Disease Assessment Scale-cognitive subscale, Korean version 
than male subjects $(n=26,39.4 \%)$ in the total ITT population. Mean (SD) ages of the patients in the naïve and switched groups were $73.59(9.43)$ and $72.20(8.50)$ years, respectively $(\mathrm{Z}=-0.801, \mathrm{p}=0.422$.). Mean (SD) durations of illness in the naive and switched groups were 17.04 (12.53) and 37.62 (21.01) months, respectively $(\mathrm{Z}=3.931, \mathrm{p}<0.001)$. Mean $(\mathrm{SD})$ scores on the CDR-SB were 6.28 (3.01) in the naïve group and 7.00 (3.41) in the switched group $(\mathrm{t}=-0.883, \mathrm{p}=0.381)$. No difference in the distribution of K-MMSE scores was detected between the naive group [19.00 (4.24)] and the switched group [18.25 (4.36)] $(\mathrm{t}=-0.803, \mathrm{p}=0.422)$, indicating no difference in the dementia severity distribution at the time of commencing treatment with galantamine. No between-group differences in any of the other demographic or baseline clinical variables were observed (Table 1).

\section{Efficacy of galantamine on cognition in combined groups}

Compared to the imputed placebo group, i.e., the natural decline estimated using the Stern equation, cognitive benefit was observed in the total sample over the 52 weeks of galantamine treatment, as evidenced by slowing cognitive decline (Table 2, Figure 2). The difference in the total score on the ADAS-cog-K between the total galantamine group and the imputed placebo group was significant at each evaluation point $(4,13,26,39$ and 52 weeks) as presented in Table 2.

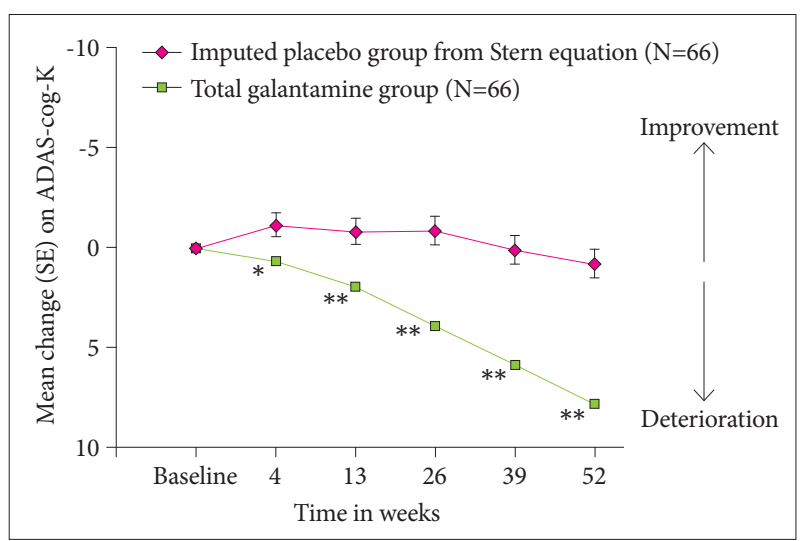

Figure 2. Distributions and patterns of changes over the 52 weeks on the ADAS-cog-K in the imputed placebo and total galantamine groups. The scores on the ordinate axis display changes from baseline ADAS-cog-K scores. The natural decline in cognition for the imputed placebo group (green, square-dotted) was estimated using the Stern equation, where initial baseline ADAS-cog-K scores of the study participants $(\mathrm{N}=66)$ were used to predict deterioration. The total galantamine group (pink, diamond-dotted) $(\mathrm{N}=66)$ showed significant therapeutic efficacy on cognition at each evaluation point (i.e., 4, 13, 26, 39, and 52 weeks), compared to the imputed placebo group. ${ }^{*} p<0.05,{ }^{* *} p<0.01$. SE: standard error, ADAS-cog-K: Korean version of the Alzheimer's Disesase Assessment Scale-cognitive subscale.

\section{Comparisons between the naïve and switched groups}

\section{Categorical primary outcome measure}

The response rates on the ADAS-cog-K at 26 weeks were $71.4 \%$ and $58.3 \%$ in the naïve and switched groups, respectively $\left(\chi^{2}=1.178, d f=1, p=0.277\right)$. The response rates at 52 weeks were not different (59.5\% for the naïve group vs. $41.6 \%$ for the switched group; $\chi^{2}=1.955, \mathrm{df}=1, \mathrm{p}=0.162$ ).

\section{Dimensional primary outcome measure}

No significant between-group differences in the change of the ADAS-cog-K score were observed at any time point (Figure 3): 4 weeks $(Z=0.415, p=1.00), 13$ weeks $(Z=0.100, p=$ 1.007), 26 weeks $(Z=-1.243, p=1.00), 39$ weeks $(Z=-1.738, p=$ 0.82), 52 weeks $(Z=-1.636, p=1.00)$. Similarly, no significant between-group differences in the changes on the ADAS-cog$\mathrm{K}$ were observed after adjusting for the difference in duration of illness using Spearman's partial correlation analysis with Bonferroni's correction. Figure 3 shows the score distribution at each evaluation point and the patterns of changes on the ADAS-cog-K in the naïve and switched groups. Furthermore, the difference on the pattern of change on the ADAS-cog-K between the naïve and switched groups over the 52 weeks was not significant (repeated-measures ANOVA, F=1.229, $\mathrm{p}=0.308)$.

\section{Secondary cognitive outcome measures}

Changes on the subset of frontal executive function and on the K-MMSE (Figure 4) were not different between the two groups. No difference in the pattern of change was observed

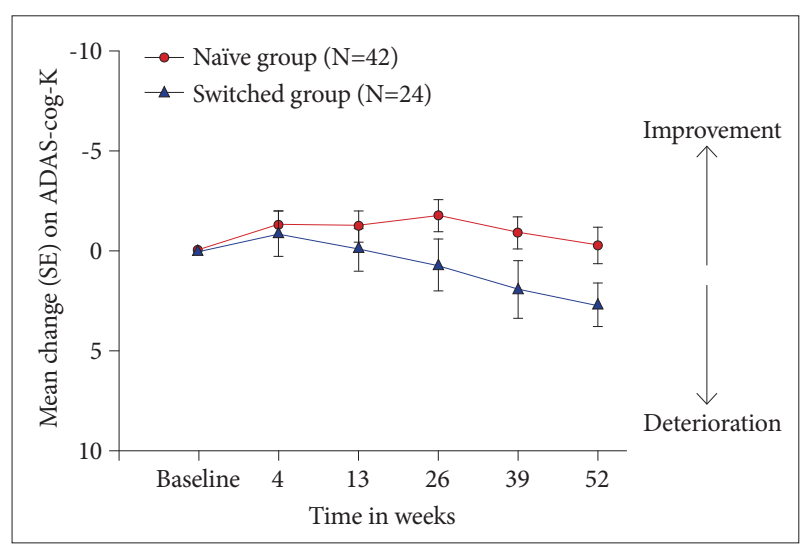

Figure 3. Distributions and the patterns of change on the ADAScog-K in the naïve and switched groups over 52 weeks. The scores on the ordinate axis display changes from baseline ADAScog-K scores. No differences in cognition were detected between the naïve group (red, circle-dotted) $(\mathrm{N}=42)$ and the switched group (blue, triangle-dotted) $(\mathrm{N}=24)$ at each evaluation point. No difference in the pattern of change on the ADAS-cog-K was observed between the naïve and switched groups over 52 weeks (repeated measures ANOVA, $F=1.229, p=0.308$ ). SE: standard error, ADAScog-K: Korean version of the Alzheimer's Disesase Assessment Scale-cognitive subscale. 
over the 52 weeks on the subset of frontal executive function (repeated measures ANOVA, $\mathrm{F}=0.687, \mathrm{p}=0.603$ ) or on the $\mathrm{K}$ MMSE (repeated measures ANOVA, $\mathrm{F}=0.383, \mathrm{p}=0.820$ ) (Figure 4).

\section{Tolerability}

Galantamine was well tolerated in both groups, and the adverse events experienced by the patients were not severe. The most common adverse events were nausea, anorexia, and indigestion. No differences in adverse events were detected between the groups.

\section{DISCUSSION}

This study investigated the efficacy of galantamine on cognition in patients with and without a history of precedent failure to respond to donepezil. We used a 52 week-prospective naturalistic study design in patients with $\mathrm{AD}$ of mild-to-moderate severity.

Because donepezil and galantamine belong to the same class of ChEI drugs, precedent failure to respond to donepezil could be hypothesized to predict that a subsequent trial of galantamine would also fail to manifest a therapeutic benefit. However, our findings show that the clinical outcomes of cognition in the group switched to galantamine after not responding to donepezil were not inferior to outcomes in the group naïe to galantamine. Thus, our findings may contribute to the evidence-based clinical approach for managing non-responding patients with $\mathrm{AD}$ of mild-to-moderate severity. Our data suggest that switching ChEI drugs is a viable clinical option. The operationally defined response rate in the switched patients was $58.3 \%$ at 6 months and $41.6 \%$ at 1 year.

We first determined that galantamine treatment in the pool- ed sample displayed significant clinical efficacy on cognition over 52 weeks. This overall analysis was made by comparing the observed declines in cognitive scores against the decline predicted for an imputed placebo-treated group, based on the Stern equation. Our finding was generally compatible with the results of previous studies which suggested improved core $\mathrm{AD}$ symptoms or slowing of the rate of cognitive decline in $\mathrm{AD}$ after at least 6 months of galantamine treatment. ${ }^{12,32,33} \mathrm{~A}$ potential limitation is that our cases had fewer years of education (mean, 9.17; SD 5.57 years) than that of cases from whom the Stern equation was derived (mean, 13.3; SD, 3.1 years). ${ }^{15}$

Following this preliminary overall analysis, we compared the effects of galantamine on cognitive decline in the naive and switched groups and found no differences in either the categorical or dimensional primary outcomes. The categorical analysis of the number of patients who showed a predefined response, i.e., improvement or no deterioration from baseline on the ADAS-cog-K score, suggests that a substantial portion of patients with mild-to-moderately severe $\mathrm{AD}$ might benefit from switching to galantamine if they have failed to respond to donepezil, regardless of previous exposure to donepezil and longer illness duration. These findings are comparable to those of previous studies that evaluated the clinical efficacy of rivastigmine in patients with mild-to-moderate $\mathrm{AD}$ who were switched from donepezil or galantamine. ${ }^{5,8}$ Our findings also are similar to a previous study conducted by Mintzer and Kershaw. Using a post-hoc analysis of a previous clinical trial with galantamine, these authors found that the clinical efficacy of galantamine was not affected by previous exposure to ChEIs. ${ }^{6}$ Furthermore, our dimensional comparisons between the naive and switched groups for the changes on the ADAS-cog-K suggest that long-term clinical benefits on cognition from switch-
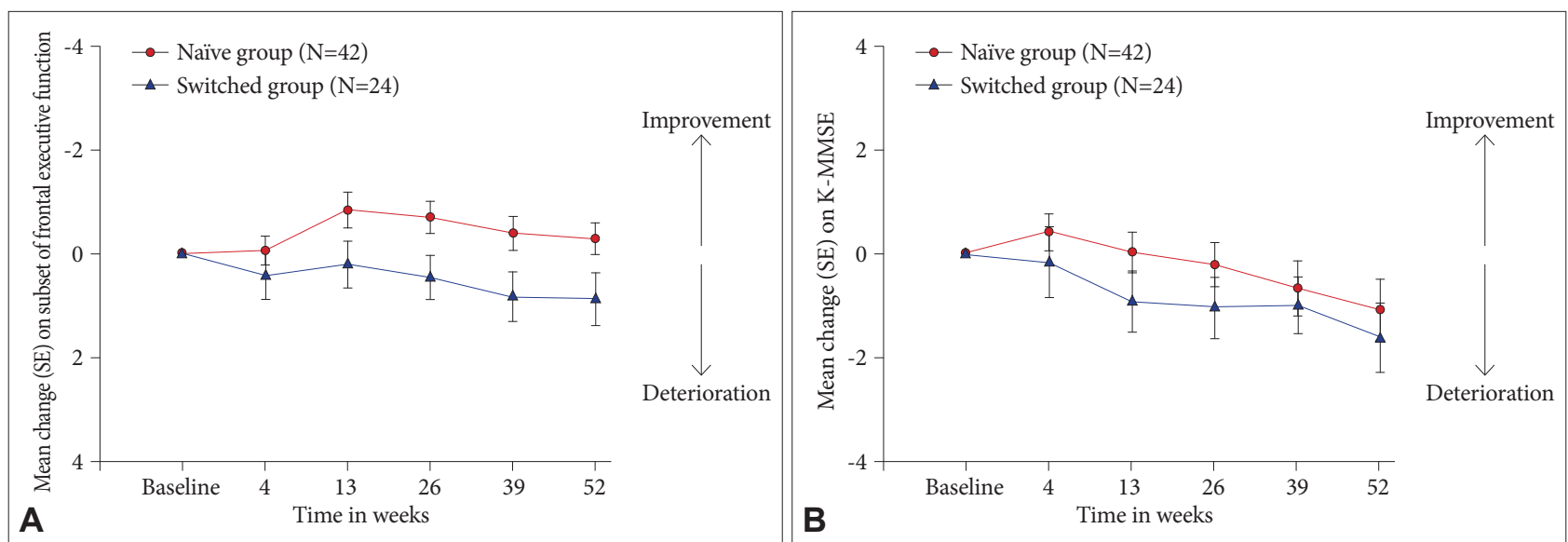

Figure 4. Changes from baseline scores on the subset of frontal executive function (A) and on the K-MMSE (B) in the naïve and switched groups over 52 weeks. No differences between the naïve (red, circle-dotted) $(\mathrm{N}=42)$ and switched groups (blue, triangle-dotted) $(\mathrm{N}=24)$ were observed for any scale at any evaluation point. No difference in the pattern of change on the subset of frontal executive function (repeated measures ANOVA, $F=0.687, p=0.603)$ or on the K-MMSE $(F=0.383, p=0.820)$ was observed between the naïve and switched groups over the 52 weeks. SE: standard error, K-MMSE: Korean Mini-Mental State Examination. 
ing to galantamine might persist over 52 weeks, regardless of previous exposure to donepezil. In addition to the ADAS-cog$\mathrm{K}$ findings, it is particularly meaningful that our switched group did not differ from the naive group in their rate of decline in frontal executive functions over the 52 weeks. This result gives convergent validity to our positive analysis based on the ADAS-cog-K scores.

The switched group in this study included only patients with mild-to-moderate $\mathrm{AD}$ who failed to respond to an initial trial of donepezil due to loss or lack of efficacy, not due to poor tolerability. Nevertheless, 58.3\% (26 weeks) and 41.6\% (52 weeks) of patients in this switched group responded to the subsequent trial of galantamine. This response rate might be modest but plausible, compared to $75 \%$ at week 12 and $44.9 \%$ at week 52 in previous studies involving galantamine. ${ }^{34,35}$ In particular, the 52-week response rate is fully comparable to those previous reports. This response rate is also in line with the report of Auriacombe et al. where $54.5 \%$ of patients who discontinued donepezil due to loss or lack of efficacy responded to rivastigmine in an open-label, 6-month trial. ${ }^{5}$

Differences in pharmacodynamic or pharmacokinetic profiles among ChEIs may partly account for the benefit of switching from donepezil to galantamine. ${ }^{9,11,36}$ These two drugs differ in their pharmacodynamics, as donepezil is a selective inhibitor of acetylcholinesterase (AChE) specific to the central nervous system, whereas galantamine is a tertiary alkaloid with dual mechanisms of action, i.e., selective inhibition of AChE over butyrylchlolinesterase and allosteric modulation of the nicotinic acetylcholine receptors (nAChR). ${ }^{37}$ Galantamine blocks the breakdown of acetylcholine by the enzyme AChE, by which it indirectly stimulates muscarinic metabotropic and nicotinic ionotropic acetylcholine receptors. The indirect muscarinic receptor agonistic effects may contribute to the improvement of cognitive function in $\mathrm{AD} .{ }^{38}$ Furthermore, the $\mathrm{nAChR}$ allosteric potentiating effect of galantamine has been associated with enhanced dopaminergic neurotransmission in the prefrontal cortex, leading to presynaptic facilitation of glutamate release, which may, in turn, contribute to improved learning and memory through N-methyl-D-aspartate receptors. ${ }^{39}$ Accordingly, the dual actions of galantamine may mediate the improvement of patients with $\mathrm{AD}$ who failed to respond to the first trial of donepezil, despite longer duration of illness.

The limitations of our study include the absence of a formal placebo group. We did not adopt a placebo control group because the local IRB expressed ethical concerns, as the clinical efficacy of ChEIs has already been proven, a placebo control group would not be approved, particularly for a 52-week longterm trial. Instead, the placebo group was imputed by modeling the natural decline estimated using the Stern equation, which is recommended for clinical trials. However, the natural deterioration course estimated from the Stern equation may have been different from the course in our Korean population, because of differences in education level and age distribution. A second limitation is that although sample size was sufficient according to our power analysis, it was relatively small and unbalanced. Third, this study adopted a LOCF analysis for the participants who did not complete the study, which might bias the analysis of the pooled sample at later time periods for comparison with the imputed placebo group based on the Stern equation because only completed cases were used in that report. However, the impact of attrition on the main analyses of this study, i.e., comparison between the naive and switched groups on the ADAS-cog-K, would not be significant considering no significant between-group differences in dropout rates. Fourth, this study included only outpatients with AD of mild-to-moderate severity. Inpatients with $\mathrm{AD}$ may have more problems with behaviors or may be different from outpatients in other demographic or clinical characteristics. So, there would be some limits to generalization of the findings of this study to all patients with $\mathrm{AD}$.

A substantial number of patients fail to respond to the first trial of any ChEI drugs recommended as the first-line pharmacological treatment for patients with mild-to-moderate AD. Despite these limitations, the results of this study suggest that switching drugs within the ChEI class is a viable option for non-responding patients with mild-to-moderate AD. Further studies should focus on 1) a prospective comparative study between patients who continue donepezil despite its ineffectiveness and those who are switched to galantamine; 2) additional studies involving different ChEI switching sequences; and 3) multicenter replication studies with larger samples.

\section{Acknowledgments}

This research was supported by the Basic Science Research Program through the National Research Foundation of Korea (NRF) funded by the Ministry of Education, Science, and Technology (NRF-2014 R1A2A1A 10052419). We thank Dr. Bernard J. Carroll, Pacific Behavioral Research Foundation, Carmel, California, USA for text editing assistance.

\section{REFERENCES}

1. Scarpini E, Scheltens P, Feldman H. Treatment of Alzheimer's disease: current status and new perspectives. Lancet Neurol 2003;2:539-547.

2. Burns A, O'Brien J, BAP Dementia Consensus group, Auriacombe S, Ballard C, Broich K, et al. Clinical practice with anti-dementia drugs: a consensus statement from British Association for Psychopharmacology. J Psychopharmacol 2006;20:732-755.

3. Wilkinson DG, Francis PT, Schwam E, Payne-Parrish J. Cholinesterase inhibitors used in the treatment of Alzheimer's disease: the relationship between pharmacological effects and clinical efficacy. Drugs Aging 2004;21:453-478.

4. Ritchie CW, Ames D, Clayton T, Lai R. Metaanalysis of randomized trials of the efficacy and safety of donepezil, galantamine, and rivastigmine for the treatment of Alzheimer disease. Am J Geriatr Psychiatry 
2004;12:358-369.

5. Auriacombe S, Pere JJ, Loria-Kanza Y, Vellas B. Efficacy and safety of rivastigmine in patients with Alzheimer's disease who failed to benefit from treatment with donepezil. Curr Med Res Opin 2002;18:129-138.

6. Mintzer JE, Kershaw P. The efficacy of galantamine in the treatment of Alzheimer's disease: comparison of patients previously treated with acetylcholinesterase inhibitors to patients with no prior exposure. Int J Geriatr Psychiatry 2003;18:292-297.

7. Bartorelli L, Giraldi C, Saccardo M, Cammarata S, Bottini G, Fasanaro $\mathrm{AM}$, et al. Effects of switching from an AChE inhibitor to a dual AChEBuChE inhibitor in patients with Alzheimer's disease. Curr Med Res Opin 2005;21:1809-1818.

8. Gauthier S, Juby A, Morelli L, Rehel B, Schecter R; EXTEND Investigators. A large, naturalistic, community-based study of rivastigmine in mild-to-moderate AD: the EXTEND Study. Curr Med Res Opin 2006; 22:2251-2265.

9. Gauthier S, Emre M, Farlow MR, Bullock R, Grossberg GT, Potkin SG. Strategies for continued successful treatment of Alzheimer's disease: switching cholinesterase inhibitors. Curr Med Res Opin 2003;19:707-714.

10. Farlow MR. Pharmacokinetic profiles of current therapies for Alzheimer's disease: implications for switching to galantamine. Clin Ther 2001;23 (Suppl A):A13-A24.

11. Maelicke A. Pharmacokinetic rationale for switching from donepezil to galantamine. Clin Ther 2001;23(Suppl A):A8-A12.

12. Raskind MA, Peskind ER, Wessel T, Yuan W. Galantamine in AD: a 6-month randomized, placebo-controlled trial with a 6-month extension. The Galantamine USA-1 Study Group. Neurology 2000;54:2261-2268.

13. Lyketsos CG, Reichman WE, Kershaw P, Zhu Y. Long-term outcomes of galantamine treatment in patients with Alzheimer disease. Am J Geriatr Psychiatry 2004;12:473-482.

14. Kawas CH, Clark CM, Farlow MR, Knopman DS, Marson D, Morris JC, et al. Clinical trials in Alzheimer disease: debate on the use of placebo controls. Alzheimer Dis Assoc Disord 1999;13:124-129.

15. Stern RG, Mohs RC, Davidson M, Schmeidler J, Silverman J, KramerGinsberg E, et al. A longitudinal study of Alzheimer's disease: measurement, rate, and predictors of cognitive deterioration. Am J Psychiatry 1994;151:390-396.

16. McKhann G, Drachman D, Folstein M, Katzman R, Price D, Stadlan EM. Clinical diagnosis of Alzheimer's disease: report of the NINCDSADRDA Work Group under the auspices of Department of Health and Human Services Task Force on Alzheimer's Disease. Neurology 1984; 34:939-944.

17. Han C, Jo SA, Jo I, Kim E, Park MH, Kang Y. An adaptation of the Korean mini-mental state examination (K-MMSE) in elderly Koreans: demographic influence and population-based norms (the AGE study). Arch Gerontol Geriatr 2008;47:302-310.

18. Lynch CA, Walsh C, Blanco A, Moran M, Coen RF, Walsh JB, et al. The clinical dementia rating sum of box score in mild dementia. Dement Geriatr Cogn Disord 2006;21:40-43.

19. Rasmusen L, Yan B, Robillard A, Dunbar F. Effects of washout and doseescalation periods on the efficacy, safety, and tolerability of galantamine in patients previously treated with donepezil: ongoing clinical trials. Clin Ther 2001;23(Suppl A):A25-A30.

20. Wilkinson DG, Howe I. Switching from donepezil to galantamine: a double-blind study of two wash-out periods. Int J Geriatr Psychiatry 2005;20:489-491.

21. Lingjaerde O, Ahlfors UG, Bech P, Dencker SJ, Elgen K. The UKU side effect rating scale. A new comprehensive rating scale for psychotropic drugs and a cross-sectional study of side effects in neuroleptic-treated patients. Acta Psychiatr Scand Suppl 1987;334:1-100.

22. Winblad B, Brodaty H, Gauthier S, Morris JC, Orgogozo JM, Rock- wood K, et al. Pharmacotherapy of Alzheimer's disease: is there a need to redefine treatment success? Int J Geriatr Psychiatry 2001;16:653-666.

23. Erkinjuntti T, Kurz A, Gauthier S, Bullock R, Lilienfeld S, Damaraju CV. Efficacy of galantamine in probable vascular dementia and Alzheimer's disease combined with cerebrovascular disease: a randomised trial. Lancet 2002;359:1283-1290.

24. Rosen WG, Mohs RC, Davis KL. A new rating scale for Alzheimer's disease. Am J Psychiatry 1984;141:1356-1364.

25. Youn JC, Lee DY, Kim KW, Lee JH, Jhoo JH, Lee KU, et al. Development of the Korean version of Alzheimer's Disease Assessment Scale (ADAS-K). Int J Geriatr Psychiatry 2002;17:797-803.

26. Mohs RC, Knopman D, Petersen RC, Ferris SH, Ernesto C, Grundman $\mathrm{M}$, et al. Development of cognitive instruments for use in clinical trials of antidementia drugs: additions to the Alzheimer's Disease Assessment Scale that broaden its scope. The Alzheimer's Disease Cooperative Study. Alzheimer Dis Assoc Disord 1997;11(Suppl 2):S13-S21.

27. Seo EH, Lee DY, Kim KW, Lee JH, Jhoo JH, Youn JC, et al. A normative study of the Trail Making Test in Korean elders. Int J Geriatr Psychiatry 2006;21:844-852.

28. Song HJ, Choi JY. A normative study of the Digit Span and the Spatial Span for the elderly Koreans. Korean J Clin Psychol 2006;25:505-532.

29. Lee JH, Kang YW, Jin JH, Na DL, Park JS. A normative study of the Korean version of Controlled Oral Word Association Test (COWAT) in the elderly. Korean J Clin Psychol 2000;19:385-392.

30. Kang YW, Na DL, Hahn SH. A validity study on the Korean Mini-Mental State Examination (K-MMSE) in dementia patients. J Korean Neurol Assoc 1997;15:300-308.

31. Folstein MF, Folstein SE, McHugh PR. "Mini-mental state”. A practical method for grading the cognitive state of patients for the clinician. J Psychiatr Res 1975;12:189-198.

32. Tariot PN, Solomon PR, Morris JC, Kershaw P, Lilienfeld S, Ding C. A 5-month, randomized, placebo-controlled trial of galantamine in $\mathrm{AD}$. The Galantamine USA-10 Study Group. Neurology 2000;54:2269-2276.

33. Takeda A, Loveman E, Clegg A, Kirby J, Picot J, Payne E, et al. A systematic review of the clinical effectiveness of donepezil, rivastigmine and galantamine on cognition, quality of life and adverse events in Alzheimer's disease. Int J Geriatr Psychiatry 2006;21:17-28.

34. Wilcock G, Howe I, Coles H, Lilienfeld S, Truyen L, Zhu Y, et al. A longterm comparison of galantamine and donepezil in the treatment of Alzheimer's disease. Drugs Aging 2003;20:777-789.

35. Jones RW, Soininen H, Hager K, Aarsland D, Passmore P, Murthy A, et al. A multinational, randomised, 12-week study comparing the effects of donepezil and galantamine in patients with mild to moderate Alzheimer's disease. Int J Geriatr Psychiatry 2004;19:58-67.

36. Maelicke A, Samochocki M, Jostock R, Fehrenbacher A, Ludwig J, Albuquerque EX, et al. Allosteric sensitization of nicotinic receptors by galantamine, a new treatment strategy for Alzheimer's disease. Biol Psychiatry 2001;49:279-288.

37. Albuquerque EX, Santos MD, Alkondon M, Pereira EF, Maelicke A. Modulation of nicotinic receptor activity in the central nervous system: a novel approach to the treatment of Alzheimer disease. Alzheimer Dis Assoc Disord 2001;15(Suppl 1):S19-S25.

38. Bodick NC, Offen WW, Levey AI, Cutler NR, Gauthier SG, Satlin A, et al. Effects of xanomeline, a selective muscarinic receptor agonist, on cognitive function and behavioral symptoms in Alzheimer disease. Arch Neurol 1997;54:465-473.

39. Schilstrom B, Ivanov VB, Wiker C, Svensson TH. Galantamine enhances dopaminergic neurotransmission in vivo via allosteric potentiation of nicotinic acetylcholine receptors. Neuropsychopharmacology 2007;32:43-53. 